\title{
Thermoelastic investigation of a servo-actuated hydraulic plastic valve undergoing a time dependant internal pressure variation
}

\author{
by F. Pezzani and A. Salerno
}

\author{
Department of Energy, Politecnico di Milano, via Lambruschini 4, 20156 Milano, Italy \\ e-mail: antonio.salerno@polimi.it
}

\begin{abstract}
This study aims at measuring the stress induced in a servo-actuated hydraulic plastic valve under pressure. Thermoelastic Stress Analysis (TSA) allowed to measure the stress distribution on the valve body and cover, when the valve was undergoing a periodic pressure variation. Previous destructive tests, carried out increasing the water static pressure inside the valve, produced a valve burst at a pressure not much higher than that, which can be reached in some real working conditions. TSA allowed spotting the points where the pressure reached particularly high values. These results will be used for the design of a new valve, which will have higher safety standards.
\end{abstract}

\section{Introduction}

Hydraulic plastic valves are light and less expensive than metallic ones, but they can withstand much lower pressures. Due to the shrinkage of the plastic material during the cooling down in the production phase, the valve walls cannot be made thicker above a certain limit. For this reason they normally present stiffening ribs on their surface. Servoactuated hydraulic valves normally present a body, a deformable membrane and a cover tightened on the body by bolts, with the deformable membrane edge clamped in between. The cover and the deformable membrane form an actuation chamber. When the valve is open, the actuation chamber is empty and the pressure of the water flowing in the valve keeps the membrane pressed against the cover. To close the valve, some water is intercepted from the upstream side and introduced in the actuation chamber between the cover and the deformable membrane. The pressure in the actuation chamber causes a deformation of the membrane, which closes the valve. Servo actuated plastic valves are widely used in agriculture, in large fields often in arid areas, where water is precious, and they have to work unattended for long periods. A braking of the valve structure would cause a large waste of water, before the valve is substituted. Different irrigation system in the world can work at different pressures, usually between 3 and 5 bar, but also up to 10 bar. The load on the valve structure produced by the water pressure is different when the valve is opened and when the valve is closed. A pressure stroke is created when the valve closes, and this can be particularly dangerous if the line working pressure is high.

Plastic valves used in irrigation have to work for years under the sun, and their surface is treated against the UV radiation, to avoid an embrittlement of their structure.

A previous destructive test made increasing the static water pressure in a plastic valve showed that the valve could burst at a pressure not much higher than that, which can be reached in some irrigation systems.

Since the production of a new valve with several technical improvements was planned, a Thermoelastic Stress Analysis (TSA) test was performed on a valve actually in production, in order to obtain indications on the stress distribution, which could help in the structural design of the new valve, to avoid dangerous stress concentrations. Plastic valves are cheap, compared to metallic ones, but their mould, which is produced by electro-erosion, can be very expensive.

\section{Thermoelastic stress analysis}

When a solid is put under stress, it undergoes a volume variation, which depends on the first stress invariant, i.e. the sum of principal stresses. If such a volume variation is fast enough, the solid undergoes an adiabatic transformation and it presents a temperature variation $\delta T$, which depends on the first stress invariant variation $\delta \sigma$ and on the thermoelastic constant $K_{0}$, according to the basic thermoelastic equation introduced by Darken and Gurry [1]:

$$
\frac{\delta T}{T_{0}}=-K_{0} \delta \sigma
$$

where $T_{0}$ is the average local temperature.

Due to heat diffusion in the material, such a temperature variation disappears with time and a constant stress is therefore not detectable. TSA needs dynamic conditions. Only a stress variation can be measured, while the average static value does not give a signal, except for some second order effects [2]. 
When a modulated load is applied, the thermoelastic effect produces a modulated temperature at the same frequency. In adiabatic conditions, the value of the local first stress invariant variation can be determined from the local amplitude of the modulated temperature, using equation (1). If the test is performed in non-adiabatic conditions however, there is a reduction of the temperature amplitude and a systematic error in the evaluation of the stress [3, 4]. This is more likely to happen in high thermal diffusivity materials with a low modulation frequency of the load applied. In these cases a correction procedure has been proposed to recover the adiabatic temperature [5].

In plastic materials, which present low thermal diffusivity, adiabatic conditions are reached at very low frequencies and no correction procedure is normally needed.

A problem more easily encountered in plastic materials is that they can work in an area where the stress - strain relation is not linear. This can lead to a wrong interpretation of the measured temperature variation.

\section{Experimental setup}

A test rig capable of producing a flow of water through the valve was prepared. The pressure variation was produced in two different ways:

1) by closing and opening the plastic valve itself with a solenoid connecting the actuation chamber with the upstream part of the valve body;

2) by closing and opening a throttle valve placed downstream of the plastic valve. black paint.

In order to have a high and homogeneous surface emissivity, the entire valve surface was painted with a matt

The tests were carried out in three days and the pressure variation was not always precisely the same. The frequency of the solenoid and of the throttle valve was comprised between $0.18 \mathrm{~Hz}$ and $0.24 \mathrm{~Hz}$. This was sufficient to have adiabatic conditions in the plastic material. Table 1 reports the pressure values and the frequencies with the two different kind of operation: solenoid and throttle valve.

\begin{tabular}{|l|c|c|c|c|c|c|}
\hline & \multicolumn{2}{|c|}{ First day } & \multicolumn{2}{c|}{ Second day } & \multicolumn{2}{c|}{ Third day } \\
\hline & $\Delta P($ bar $)$ & Frequency $(\mathrm{Hz})$ & $\Delta P($ bar $)$ & Frequency $(\mathrm{Hz})$ & $\Delta P($ bar $)$ & Frequency $(\mathrm{Hz})$ \\
\hline Solenoid & 3.8 & 0.21 & 3 & $0.18-0.24$ & $3-3.05$ & $0.18-0.24$ \\
\hline Throttle valve & 3.8 & 0.21 & 3.2 & 0.21 & - & - \\
\hline
\end{tabular}

IR images were acquired using a IR Cedip Titanium camera, with a InSb focal plane array $320 \times 256$ and a NETD of $25 \mathrm{mK} @ 30^{\circ} \mathrm{C}$. The reference signal for the lock-in analysis was taken from a strain gauge placed on the valve body. After the test some parts of the valve were cut and some rectangular samples produced to experimentally obtain the value of the thermoelastic constant. Imposing a load on a sample of known cross section and measuring the temperature $T_{0}$ and the temperature variation $\Delta T$, the thermoelastic constant is readily evaluated from Eq. 1 .

The cover and the valve body were made of different plastic materials, therefore two different thermoelastic constants were obtained and used to interpret the results.

Fig. $1 \mathrm{~A}$ and $\mathrm{B}$ show the experimental set up during the test and the valve cover.

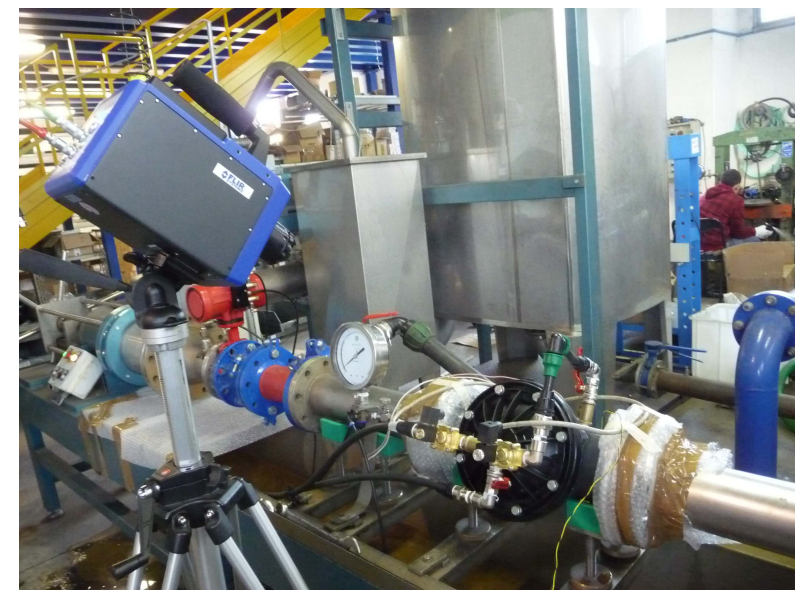

Fig.1 A. Experimental set up during the test.

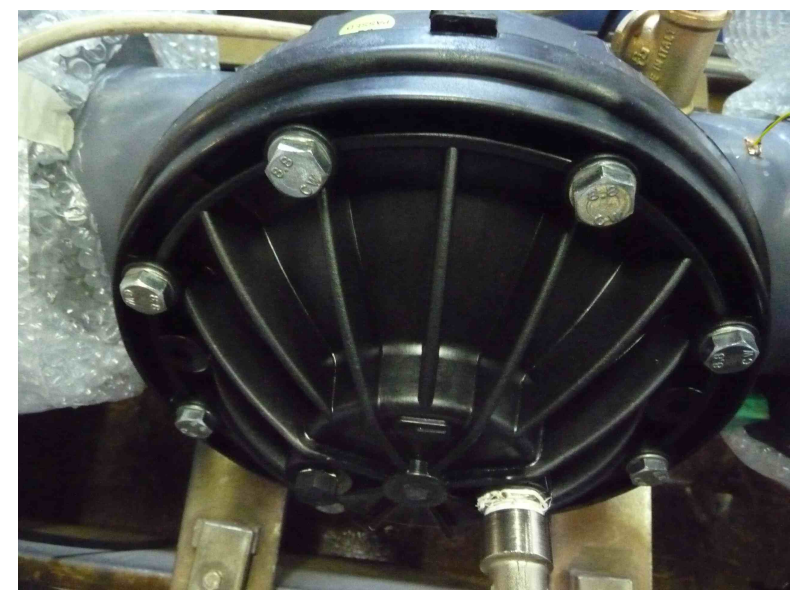

Fig.1 B. Valve cover.

For a TSA measurement a pressure modulation is necessary, because a static pressure does not give any thermoelastic signal. The normal valve operation is through a solenoid acting on the valve itself, and the pressure is only increased in the actuation chamber under the cover and in the upstream part of the valve body.

The throttle valve disposed downstream increased the pressure in the whole valve. This second way of loading the valve closing and opening the flow downstream with a throttle valve was aimed at studying the stress in the valve body 
when water flows through the open valve mounted in a pipeline working at a given pressure. The stress distribution created by such a static pressure can be revealed using the thermoelastic effect only by creating a pressure modulation.

\section{Experimental results}

The experimental tests can be divided in tests in which the valve was operated by the solenoid and tests in which the pressure in the whole valve was controlled by the throttle valve disposed downstream.

\subsection{Operation with the solenoid}

Fig. 2 shows the cover of the valve operated with the solenoid, with $\Delta P=3$ bar and $f=0.21 \mathrm{~Hz}$. In this test when the valve is closed the water fills the actuation chamber under the cover and half of the valve body. The stress is concentrated at the centre of the cover and at the ending points of some ribs.

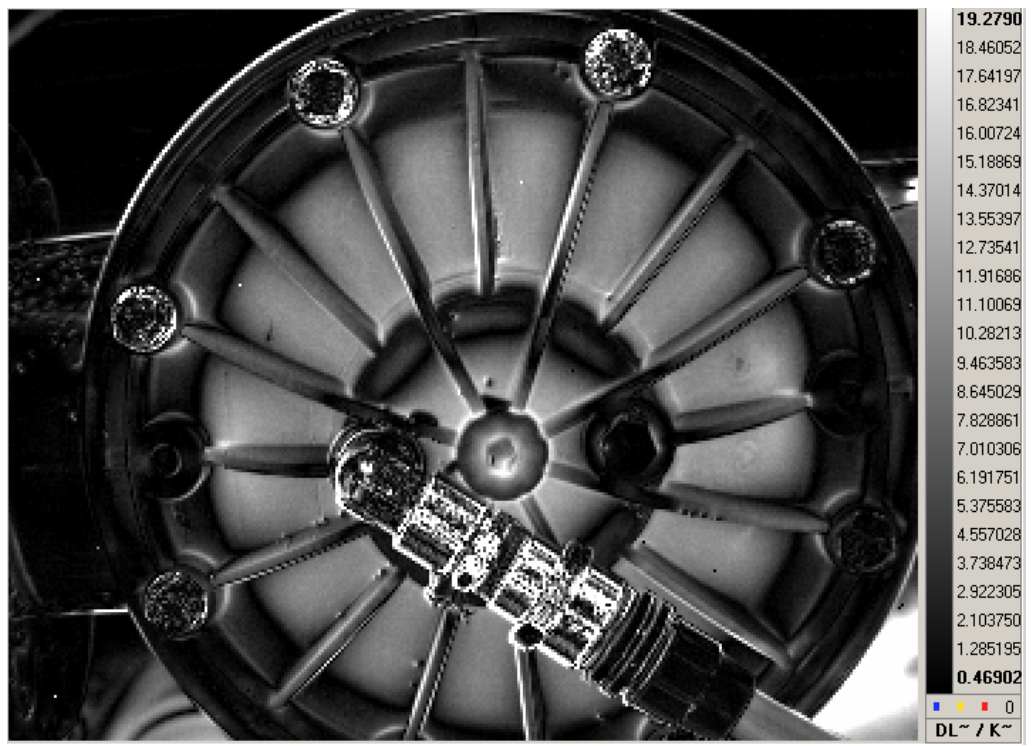

Fig.2. TSA image of the cover, when the valve is operated by the solenoid ( $\triangle P=3 \mathrm{bar})$.

Fig. 3 and Fig 4 , taken at $\Delta P=3.8$ bar and $f=0.21 \mathrm{~Hz}$, show two different parts of the cover, where the stress reached the highest values.

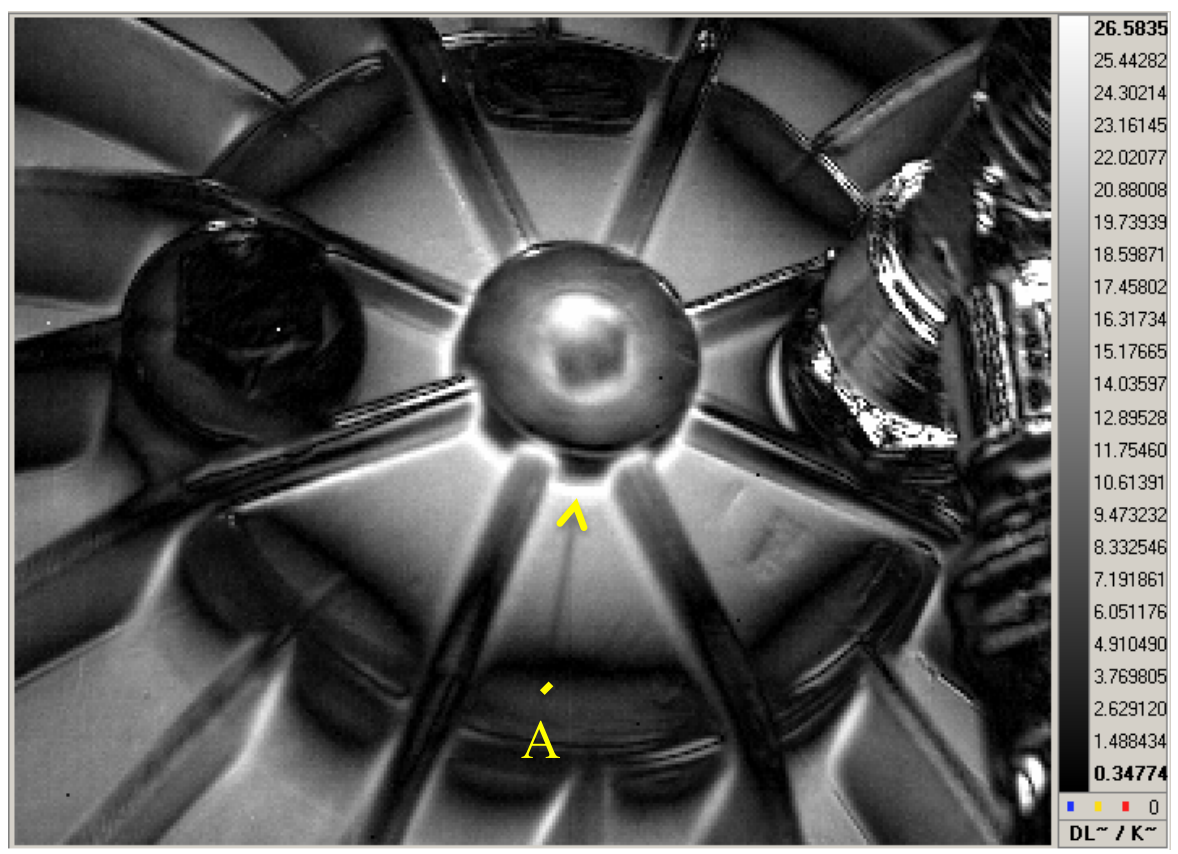

Fig.3. Central part of the cover, when the valve is operated by the solenoid ( $\triangle P=3.8$ bar). 


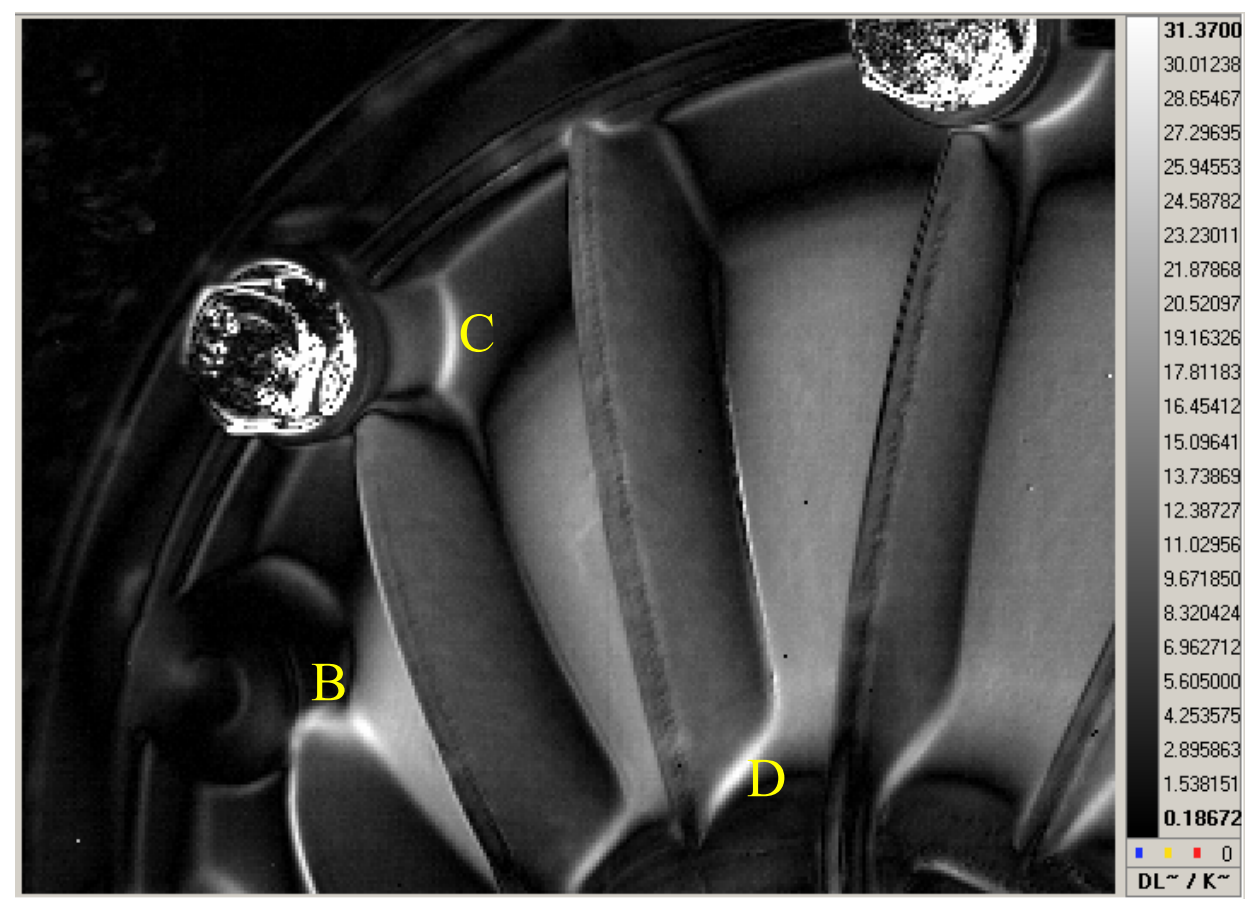

Fig.4. TSA image of some ribs on the cover, when the valve is operated by the solenoid ( $\triangle P=3.8 \mathrm{bar})$.

The highest stress values were reached in correspondence of points $\mathrm{A}=33,6 \mathrm{MPa}, \mathrm{B}=33 \mathrm{MPa}, \mathrm{C}=29.7 \mathrm{MPa}$ and $\mathrm{D}=32.7 \mathrm{MPa}$ (Figs. 3 and 4).

Fig. 5 shows the body of the valve from below, when it is operated by the solenoid. The water is arriving from the right side and the left part of the body remains empty when the valve is closed.

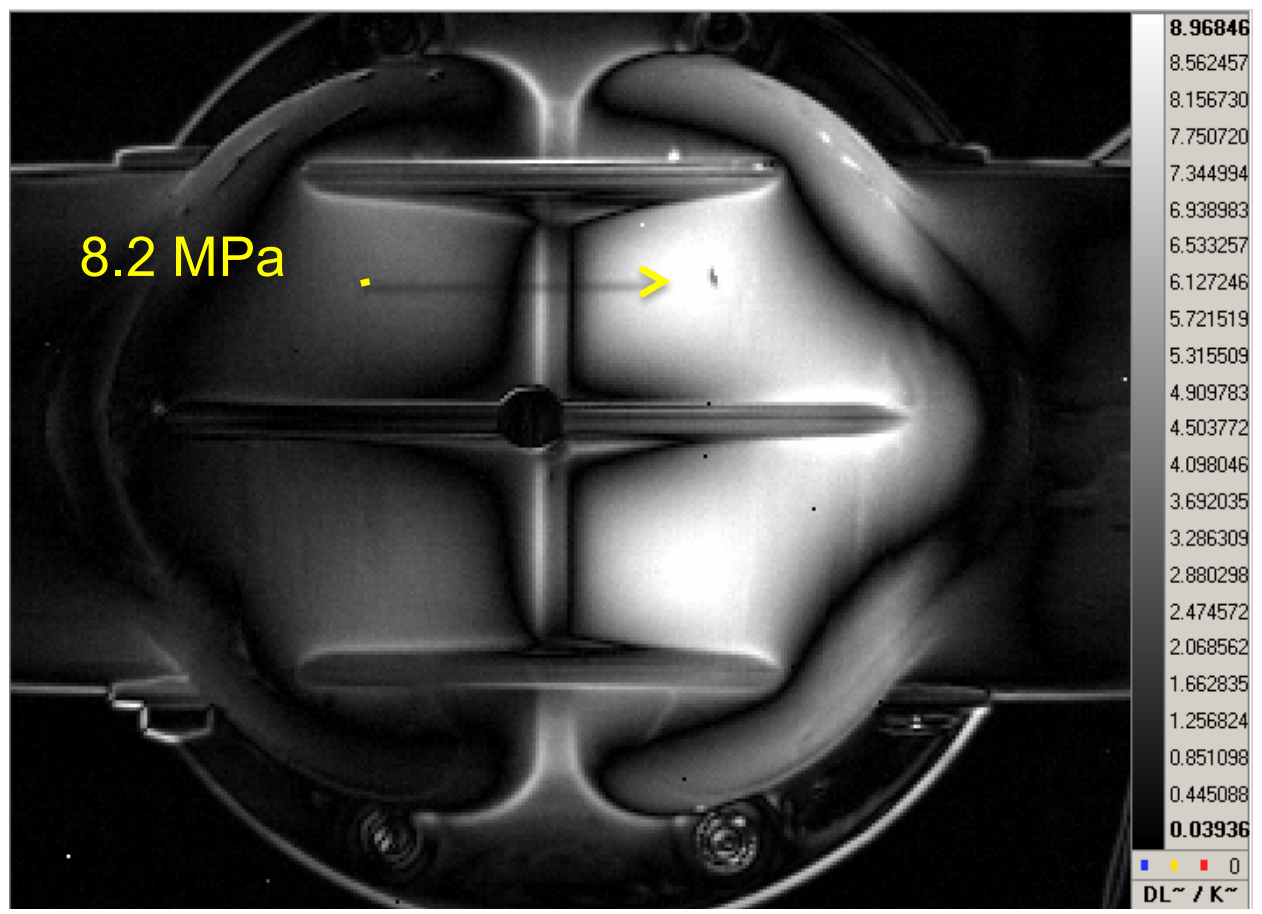

Fig.5. TSA image of the valve body from below, when the valve is operated by the solenoid ( $\triangle P=3.8$ bar).

The stress variation on the right part of the valve body is clearly higher, because this is the upstream side, where the water remains when the valve is closed. Stress values are however much lower than on the cover. Also the plastic material is different from that used for the cover. 


\subsection{Operation with the throttle valve}

In the operation with the throttle valve, the actuation chamber formed between the flexible membrane and the cover is empty and the membrane is bended outwards against the internal part of the cover by the pressure of the water flowing inside. Fig. 6 shows the TSA result on the cover in this case.

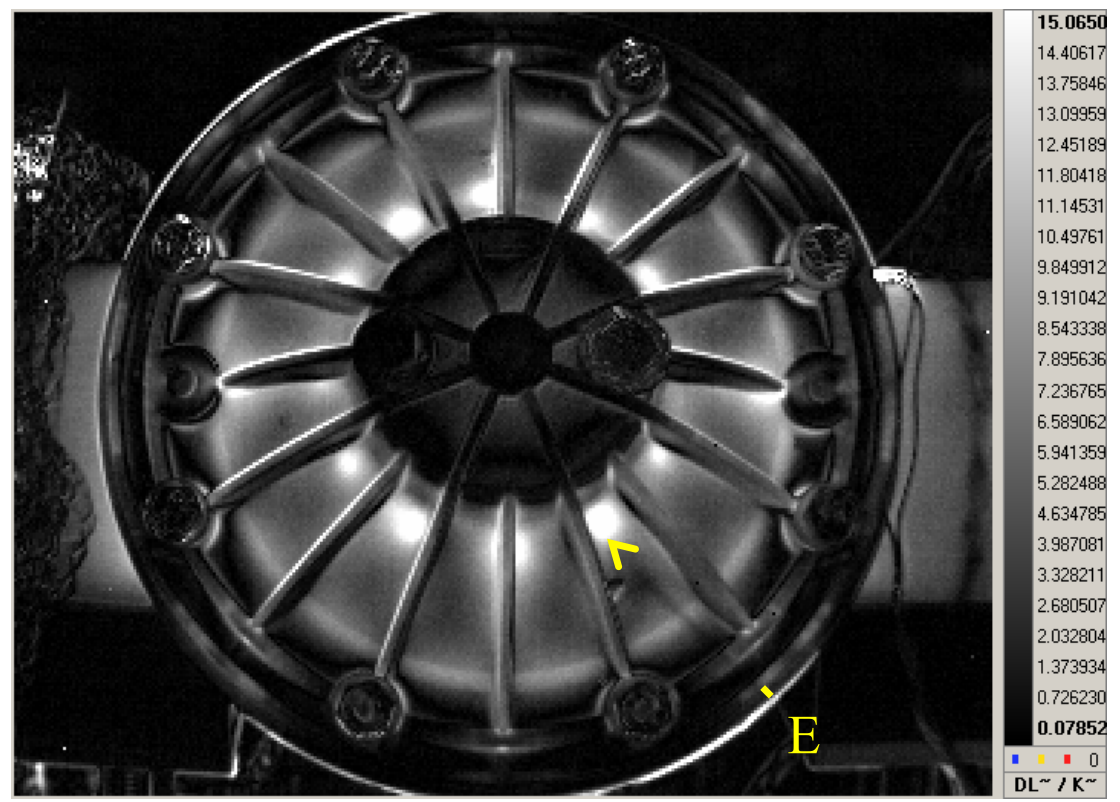

Fig.6. TSA image of the cover, when the valve is operated by the throttle valve positioned downstream ( $\Delta P=3.2 \mathrm{bar})$.

The presence of the flexible membrane between the water flowing inside the valve and the cover produces a much lower stress of the central part of the cover and a higher stress of the region around the central part. The central part of the cover forms a step that causes a further deformation of the elastic membrane, which partially counter-reacts to the water pressure, lowering the stress in the central part of the cover and increasing it at the step edge.

At the centre of the cover the stress reaches a maximum of $5.8 \mathrm{MPa}$, while on point $\mathrm{E}$ it reaches $27.5 \mathrm{MPa}$.

In Fig. 7 is reported a detail of the cover.

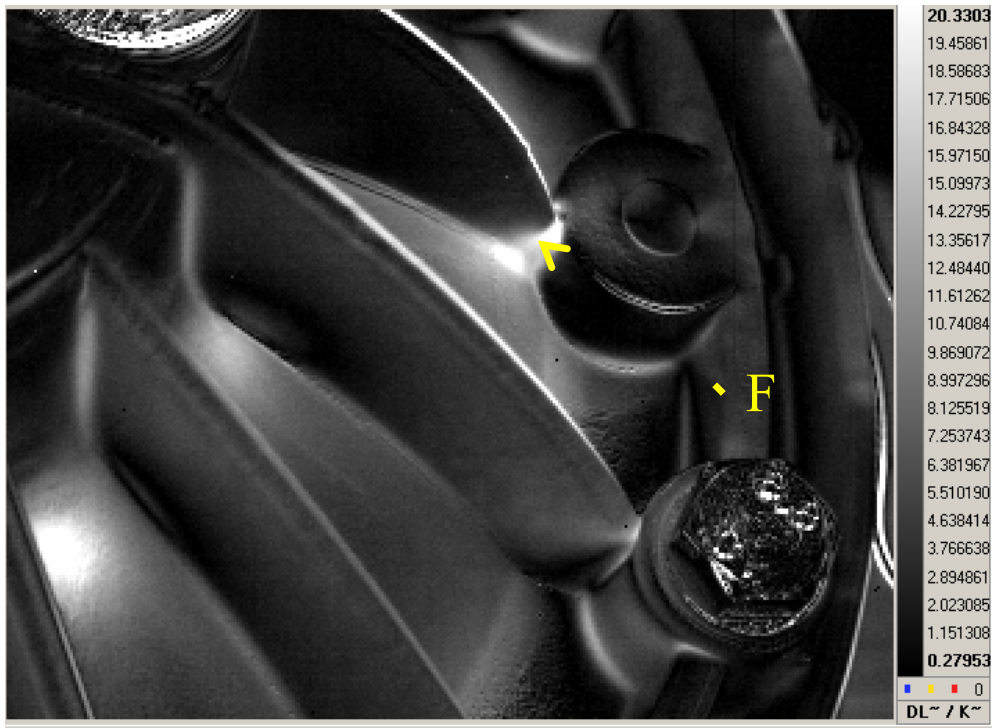

Fig.7. TSA image of part of the cover: the stress in $\mathrm{F}$ is $30.6 \mathrm{MPa}(\Delta P=3.2 \mathrm{bar})$.

A structurally critical point is point $\mathrm{F}$ at the rib end, where the maximum stress value of $30.6 \mathrm{MPa}$ is reached. Considering that this value was obtained for a $\Delta P=3.2 \mathrm{bar}$, with a linear extrapolation this would correspond to a value of 36.3 $\mathrm{MPa}$ for a $\Delta P=3.8$ bar. This means that point $\mathrm{F}$ in this configuration (operation by the throttle valve) presents a stress 
concentration, which is higher than point A of Fig. 3. In particular this rib and its symmetrical is shorter at its end than the other ribs and this causes a higher localized stress.

\section{Conclusions}

A thermoelastic study was conducted on a servo-actuated hydraulic plastic valve using two different ways of cyclic loading: opening and closing the valve with a solenoid, and opening and closing the downstream flow with a throttle valve. The stress induced in the valve is realistic in both cases, the first simulating the working conditions when the valve is closed, with the water filling the actuation chamber and the upstream side of the body, the second simulating the effect of the pressure when the valve is open and mounted in a pressurized pipeline.

The stress distribution in the two cases is very different and the TSA allowed spotting the most critical points, in particular around the center of the cover where a step is present and at the end of two of the reinforcing ribs, presenting a smaller cross section.

The results obtained allowed for an improvement of the structural design of a new kind of plastic valve, which will be soon put into production.

\section{REFERENCES}

[1] L. Darken and R. Gurry, Phisical Chemistry of Metals, McGraw-Hill, London, 1953

[2] A. K. Wong, R. Jones, J.G. Sparrow, Thermoelastic constant or thermoelastic parameter? J Phys Chem Solid 48(8), pp.749-753, 1987

[3] M. H. Belgen, Infrared Radiometric Stress Instrumentation Application Range Study, NASA CR-1067, 1968

[4] S. Offermann, J. L. Beaudoin, C. Bissieux, and H. Frick, Thermoelastic Stress Analysis Under Nonadiabatic Conditions, Experimental Mechanics, 37, pp. 409-413, 1997

[5] A. Salerno, S. Desiderati, Procedure proposal for the correction of nonadiabatic thermoelastic stress analysis results, Rev. Sci. Instrum., 75, pp. 507-514, 2004 